\title{
BMJ Open Trunk muscle exercises as a means of improving postural stability in people with Parkinson's disease: a protocol for a randomised controlled trial
}

\author{
Ryan P Hubble, ${ }^{1}$ Geraldine A Naughton, ${ }^{2}$ Peter A Silburn, ${ }^{3}$ Michael H Cole ${ }^{1}$
}

To cite: Hubble RP, Naughton GA, Silburn PA, et al. Trunk muscle exercises as a means of improving postural stability in people with Parkinson's disease: a protocol for a randomised controlled trial. BMJ Open 2014:4:e06095

doi:10.1136/bmjopen-2014006095

- Prepublication history for this paper is available online. To view these files please visit the journal online (http://dx.doi.org/10.1136/ bmjopen-2014-006095).

Received 14 July 2014 Revised 16 October 2014 Accepted 25 November 2014

\section{CrossMark}

${ }^{1}$ School of Exercise Science, Australian Catholic University, Brisbane, Australia

${ }^{2}$ School of Exercise Science, Australian Catholic University, Melbourne, Australia

${ }^{3}$ The University of

Queensland, Centre for

Clinical Research, Brisbane,

Australia

Correspondence to

Dr Michael H Cole;

michael.cole@acu.edu.au

\section{ABSTRACT}

Introduction: Exercise has been shown to improve clinical measures of strength, balance and mobility, and in some cases, has improved symptoms of tremor and rigidity in people with Parkinson's disease (PD). However, to date, no research has examined whether improvements in trunk control can remedy deficits in dynamic postural stability in this population. The proposed randomised controlled trial aims to establish whether a 12-week exercise programme aimed at improving dynamic postural stability in people with PD; (1) is more effective than education; (2) is more effective when training frequency is increased; and (3) provides greater long-term benefits than education. Methods/design: Forty-five community-dwelling individuals diagnosed with idiopathic PD with a falls history will be recruited. Participants will complete baseline assessments including tests of cognition, vision, disease severity, fear of falling, mobility and quality of life. Additionally, participants will complete a series of standing balance tasks to evaluate static postural stability, while dynamic postural control will be measured during walking using head and trunk-mounted threedimensional accelerometers. Following baseline testing, participants will be randomly-assigned to one of three intervention groups, who will receive either exercise once per week, exercise 3 days/week, or education.

Participants will repeat the same battery of tests conducted at baseline after the 12-week intervention and again following a further 12-week sustainability period.

Discussion: This study has the potential to show that low-intensity and progressive trunk exercises can provide a non-invasive and effective means for maintaining or improving postural stability for people with PD.

Importantly, if the programme is noted to be effective, it could be easily performed by patients within their home environment or under the guidance of available allied health professionals.

Trial registration number: The protocol for this study is registered with the Australian New Zealand Clinical Trials Registry (ACTRN12613001175763).

\section{INTRODUCTION}

Prospective studies indicate that the incidence of falls are much greater for people

\section{Strengths and limitations of this study}

- This study has been designed as a randomised controlled trial, which is currently considered the best methodological approach for evaluating the efficacy of a specific intervention.

- The proposed study will be the first to assess whether dynamic postural stability during walking can be improved or maintained in people with Parkinson's disease who regularly perform specific exercises to improve trunk mobility and endurance.

- This study seeks to assess changes in static and dynamic balance using continuous measures rather than graded clinical tests that are based on Likert scales, as these may be more sensitive for detecting improvements in postural stability for this patient group.

- While it would be important to examine whether improvements in postural stability are associated with a reduction in falls, the large sample size required to achieve this goal (approximately 120 participants per group) is prohibitive.

- Owing to the nature of the chosen intervention, the findings may only be applicable to patients who experience mild-to-moderate symptoms and are healthy enough to perform the exercises. As such, alternate interventions may be necessary for individuals who present with more advanced symptoms.

with Parkinson's disease (PD) than for agematched controls, with up to $68 \%$ of people with PD falling at least once each year and up to $50 \%$ of these individuals experiencing recurrent falls. ${ }^{12}$ The increased falls risk in this population is compounded by an increased risk of injury, as differences in the postural responses of people with PD place them at a greater risk of sustaining a significant fall-related injury than age-matched controls. ${ }^{3}$ Falls and fall-related injuries often lead to a fear of falling, reduced mobility, poorer muscle strength and loss of independence, all of which ultimately influence 
an individual's mortality, morbidity and quality of life. ${ }^{4}$ Biomechanical research involving healthy younger adults ${ }^{5}$ has shown that the trunk segment plays an important role in modulating gait-related oscillations and maintaining head stability; an important goal of the human postural control system. However, the increased axial rigidity that is evident in people with $\mathrm{PD}^{6}$ significantly impairs the trunk's capacity to attenuate these movement-related forces, which inadvertently reduces head stability and impairs the clarity of the visual and vestibular information used in balance control. In the early stages of the disease, the symptoms of PD are typically managed using any number of antiparkinsonian medications. However, these medications are unfortunately not always effective at improving symptoms of axial rigidity ${ }^{6}$ and often lead to undesirable side effects including dopamine-induced dyskinesias or motor fluctuations that have the potential to increase the risk of falls in people with PD. As such, there is a clear need for alternative therapies that can be easily implemented, have low running costs and have the potential to improve postural control, segmental mobility and falls risk in this population.

It is important for individuals to be able to effectively control their body's segments to maintain postural stability and limit the risk of falling during both static and dynamic activities of daily living. Older adults demonstrate poorer postural stability during tasks requiring dynamic postural control (eg, walking and turning), which can place them at an increased risk of falling. ${ }^{7}$ Age-related declines in dynamic postural control may be further exacerbated with the presence of PD, which would exacerbate the decreased balance and higher falls rate evident in this population. ${ }^{189}$

Given that the head and trunk comprise $60 \%$ of the overall mass of the body ${ }^{10}$ it seems reasonable to suggest that one's ability to precisely coordinate trunk movements would contribute significantly to attenuating movement-related oscillations and maintaining postural stability during these activities. An examination of segmental stability for different regions of the upper body in a healthy population showed that trunk movements were smaller than those of the head and neck during walking. ${ }^{11}$ However, separate research suggests that the trunk has a more irregular movement pattern than the head during gait. ${ }^{5}$ The authors argued that the trunk may serve to attenuate forces during dynamic tasks to stabilise the head, and preserve the quality of the visual and vestibular feedback required for postural control. If an individual has increased axial rigidity ${ }^{6}$ and is unable to adequately control the trunk segment during dynamic tasks, then the exaggerated movements of the trunk may have a direct impact on head stability and overall balance.

A common method used to evaluate head and trunk stability during dynamic tasks is the harmonic ratio (HR), which provides a measure of the stability of gait-related accelerations by evaluating the stride-to-stride regularity of the harmonics within the acceleration signal. ${ }^{12}$ Walking patterns that produce higher HRs will be characterised by a more regular acceleration profile over successive gait cycles (ie, less stride-to-stride variability); hence, the gait pattern is deemed to be more stable. ${ }^{13}$ People with PD who fall are known to have increased mediolateral (ML) and anteroposterior (AP) movements of the trunk during sitting, ${ }^{14}$ less regular pelvic movements (lower HRs) ${ }^{15}$ and increased ML head movement during gait. ${ }^{16}$ Collectively, these studies suggest that some of the falls experienced by people with PD may be related to a reduced capacity for these individuals to adequately coordinate the body's segments during dynamic tasks. As such, there is a clear need to evaluate the efficacy of different non-invasive interventions aimed at maintaining and/or improving trunk mobility and control to improve postural stability in this population. To date, few studies have investigated the efficacy of different non-invasive methods for improving balance and reducing falls risk in this high-risk population. ${ }^{17-21}$

It is widely recognised that exercise is an effective means of maintaining or improving cardiovascular and musculoskeletal health, both of which are critical for preserving physiological functioning and independence. Furthermore, some modes of exercise have been shown to be effective at improving standing balance, ${ }^{22}{ }^{23}$ symptoms of anxiety and depression ${ }^{24} 25$ and reducing fall rates $^{26}$ and risk of falling ${ }^{22} 27$ in otherwise healthy individuals. A number of previous studies have also provided evidence to support the short-term benefits of exercise for improving clinical measures of mobility, ${ }^{15} 17 \quad 28-30$ postural stability, ${ }^{15} 17{ }^{28-30}$ quality of life, ${ }^{31}$ cognitive function $^{31} 32$ and symptom severity in people with $\mathrm{PD}^{29} 30$ Current evidence suggests that when programmes include more challenging balance exercises, they may offer greater benefits for balance and mobility. ${ }^{17}$ For example, tai chi is a specific form of exercise known to challenge the balance system. Previous research has shown tai chi can improve measures of static postural stability in people with PD. ${ }^{33}$ However, it is important to note that the results of a recent systematic review suggest that other forms of exercise may also provide similar benefits to balance in this population. ${ }^{34}$

While this systematic evidence supports that exercise improves clinical measures of balance, mobility and disease severity, many of the improvements did not achieve a level that would be considered a minimally clinically important change. Furthermore, most of the balance and mobility assessments used in previous studies have relied on Likert scales to assess function, which may limit their ability to discriminate between people with PD who fall and those who do not. As such, it is possible that the incorporation of biomechanical measures of dynamic postural stability may improve our capacity to accurately detect improvements or declines in balance for this population, which would facilitate better identification of patients who are at a higher risk of falling. However, the investigators are unaware of any 
previous research that has investigated whether exercise can improve quantitative measures of dynamic postural stability in people with PD. A possible explanation for this may be that such a study would require the use of complex measuring equipment that is typically only available in a laboratory setting, making it a higher order of investigation and difficult to assess in a clinical environment.

As such, the proposed randomised controlled trial aims to establish whether a 12-week exercise programme aimed at improving dynamic postural stability in people with PD; (1) is more effective than education; (2) is more effective when training frequency is increased; and (3) provides greater long-term benefits than education. It is hypothesised that the both exercise programmes will improve dynamic postural stability more than education, however training at an increased frequency will yield better improvements for the people with PD.

\section{METHODS}

The proposed randomised controlled trial will be conducted in 2014/2015 and seeks to improve the mobility and endurance of the trunk and its supporting musculature. This study protocol was developed in accordance with the Consolidated Standards of Reporting Trials (CONSORT) guidelines. ${ }^{35}$

\section{Participants}

Forty-five participants diagnosed with idiopathic PD, based on the UK Brain Bank Criteria ${ }^{36}$ and who have a history of two or more near-misses and/or one fall or more in the previous 12 months will be recruited from: (1) neurology clinics, (2) community support groups, (3) and a pre-existing database of people with PD who have expressed an interest in participating in research. Prospective participants will be sent an information letter outlining the details of the study and inviting them to contact a member of the research team if they are interested in participating in the research. On contacting a member of the research team, prospective participants will be screened to ensure that they all meet the requirements of the study and, if they are deemed eligible for inclusion, a time will be scheduled to conduct the baseline assessments. Participants will be excluded if they: (1) are unable to stand and walk independently without the use of a walking aid, (2) have any significant visual (Bailey-Lovie high-contrast visual acuity $>0.30 \log$ MAR) or cognitive impairment (Addenbrooke's cognition examination score $<82$ ), (3) have uncontrolled hypertension, (4) are taking psychotropic medications, (5) have any significant limitations due to osteoporosis, (6) have had any orthopaedic surgery within the previous year, (7) have any serious neck, shoulder or back injuries; including spinal fusions, or (8) have received deep brain stimulation surgery to manage their symptoms. For the purposes of this study, a fall will be defined as 'any coming to the ground or lower level not as the result of a major intrinsic event or overwhelming hazard' and a near miss will be defined as 'an event on which an individual felt that they were going to fall but did not actually do so'. ${ }^{18}$ All volunteers will be asked to provide written informed consent in accordance with the Declaration of Helsinki prior to participation in the study.

To determine a suitable sample size, a power calculation was completed based on the HR, the primary measure of this study. The sample size was calculated using ML head accelerations from a previous study that assessed differences in dynamic postural stability in PD compared with healthy controls using the HR. ${ }^{37}$ On the basis of this calculation, it was concluded that a minimum of 11 participants per group is needed to confidently report any significant changes in dynamic postural stability (diff $=0.05, \quad \mathrm{SD}=0.04$, Cohen's $\mathrm{d}=1.25$, Power $=80 \%, p=0.05$ ). Given the longitudinal nature of the research, 15 individuals will be recruited per intervention group to accommodate a $25 \%$ rate of attrition. The experimental procedures for this study have been approved by the Australian Catholic University Human Research Ethics Committee. To ensure participants are assessed under similar conditions during each testing session, all procedures will be scheduled to start within 1-2 $\mathrm{h}$ of the participants taking their medication. This will ensure the participants are comfortable and safe during the assessments and that the results are representative of how the individuals might perform such tasks in the real world.

\section{Clinical measures}

Individuals who provide consent to participate in this study will be asked to attend an initial session at the Australian Catholic University (Brisbane) during which a series of baseline assessments will be performed. This battery of tests will include clinical assessments of: (1) cognitive function (Addenbrooke's Cognitive Examination $(\mathrm{ACE})),{ }^{38}$ (2) visual acuity (Bailey-Lovie high-contrast visual acuity ${ }^{39}$ ), (3) disease severity (Unified Parkinson's Disease Rating Scale (UPDRS), the modified Hoehn \& Yahr (H\&Y) scale, ${ }^{40}$ the Schwab \& England Activities of Daily Living Scale $^{41}$ and the PD Gait and Falls Questionnaire (PD-GFQ $\left.)^{42}\right), \quad(4)$ fear of falling (Activity-specific Balance Confidence Scale ${ }^{43}$ ), (5) mobility (Timed Up and $\mathrm{Go}^{44}$ ) and (6) quality of life (Parkinson's disease questionnaire 39 (PDQ-39)). ${ }^{45}$ The PD-GFQ is a 16 -item tool that assesses the extent of any falls and gait difficulties experienced by people with PD and incorporates six questions that are summed to give the freezing of gait (FOG) score. ${ }^{42}$ The ACE was selected to assess cognitive function, as it incorporates the Mini Mental State Examination and has been shown to have high sensitivity and specificity for detecting dementia (cut-off $<82$ gives $82 \%$ sensitivity and $100 \%$ specificity). The other assessments were selected as they have been shown to be both reliable and valid, ${ }^{38} 46-49$ and have been used previously to assess individuals with PD. ${ }^{15} 50$ 


\section{Postural stability measures}

To evaluate dynamic postural stability, participants will be asked to walk along a $10 \mathrm{~m}$ walkway at a comfortable self-selected pace for four trials and will be offered a rest break between trials to minimise the risk of fatigue. While completing this task, movement patterns of the head and trunk will be measured using two microelectromechanical system three-dimensional accelerometers (Noraxon Inc, Scottsdale, Arizona, USA) sampling at a rate of $500 \mathrm{~Hz}$. Prior to testing, the accelerometers will be statically calibrated using the methods described previously. ${ }^{51}$ Calibration involves aligning each sensing axis of the accelerometer perpendicular to a horizontal surface to determine a conversion factor that describes gravitational acceleration (1 gravitational unit or $1 \mathrm{~g}$ ). Following static calibration, an accelerometer will be firmly attached over the occipital protuberance of the skull via a sport headband and another will be attached directly to the skin using double-sided tape over the spinous process of the 10th thoracic vertebra (T10). To detect gait events, such as heel strike and toe off during the gait cycle, two pressure-sensitive footswitches (Noraxon Inc) will be placed bilaterally under the calcaneus, the distal end of the first phalange and the distal end of the first and fifth metatarsals of the foot.

Static postural stability will be assessed while participants are standing quietly on a portable force plate that is sampling data at an effective rate of $200 \mathrm{~Hz}$ (Advanced Mechanical Technology Inc, USA). Participants will complete two $30 \mathrm{~s}$ trials that will involve standing as still as possible for each of the following conditions: (1) on a firm surface with eyes open, (2) on a firm surface with eyes closed, (3) on a foam surface with eyes open and (4) on a foam surface with eyes closed. Before start of each trial, participants will be asked to look straight ahead at a cross that will be placed on the wall at eye level with their arms resting at their sides and their feet $10 \mathrm{~cm}$ apart. Measurements derived from the force plate data will include: peak RMS displacement of the centre of pressure and postural sway velocity in the $\mathrm{AP}$ and ML directions.

In addition to the acceleration profiles that will be collected for the head and trunk, muscle activation patterns for the thoracic and lumbar erector spinae will be measured at $1500 \mathrm{~Hz}$ using a wireless Noraxon surface electromyography (EMG) system (Noraxon Inc). In healthy individuals, the erector spinae muscles show a phasic increase in activation just after heel-contact to counter forward trunk flexion during walking. ${ }^{52}$ The erector spinae muscles were chosen for evaluation because individuals with PD are known to have decreased trunk muscle performance than age-matched controls, ${ }^{53}$ which may influence their capacity to control trunk motion during walking. Prior to applying the surface electrodes over the muscles of interest, the skin will be prepared with an abrasive gel (Nuprep; Weaver Company, Aurora, Colorado, USA), and then cleaned thoroughly with an isopropyl alcohol wipe to minimise impedance at the electrode-skin interface and improve clarity of the myoelectric signal. ${ }^{54}$ For individuals with excessive hair over the muscles of interest, the area will be shaved in order to maximise the fidelity of the myoelectric signal and ensure the best possible adherence to the skin. After skin preparation, four pairs of $\mathrm{Ag} / \mathrm{AgCl}$ pregelled surface electrodes (AMBU Blue Sensor, Ballerup, DK; $34 \mathrm{~mm}$ diameter, $10 \mathrm{~mm}^{2}$ sensing area) will be placed with a centre-to-centre interelectrode distance of $34 \mathrm{~mm}$. Specifically, these electrode pairs will be placed bilaterally $5 \mathrm{~cm}$ lateral to the spinous process of the T10 vertebral body and $2 \mathrm{~cm}$ lateral to the spinous process of the third lumbar (L3) vertebral body. ${ }^{55}$

To facilitate comparisons between the different testing dates and the different participant groups, the EMG data will be normalised to the muscle activity levels recorded for the participants during a maximal voluntary contraction (MVC) of the erector spinae. To perform the MVC, the participants will lie prone/prostrate on a padded table with their hips flexed and their feet on the floor. The participant will then be asked to complete three practice trials to learn the movement before performing three maximal efforts that involve simultaneously extending both hips to raise the legs to a horizontal position to activate the erector spinae muscle group. A restraining force will be applied to the legs of the participants to make sure that their legs remain horizontal $\left(180^{\circ}\right)$ while performing the test to produce the MVC. This method was chosen in preference to the traditional Biering-Sorensen test, due to the potential difficulties that older participants may have with this movement. ${ }^{55}$

All data collection will be performed using the MyoResearch XP software to ensure that the data from the different systems remain synchronised. Participants will be re-tested using the assessments outlined above: (1) after the 12-week intervention to establish the immediate effects of the exercise programme on postural stability and (2) 12 weeks after the completion of the intervention to evaluate the retention of any benefits over the longer term (ie, 24 weeks following baseline). The battery of assessments and the time points at which they will be taken are summarised in table 1 and the flow of recruitment, data collection and follow-up procedures are outlined in figure 1 .

\section{Data analyses}

Data from the raw accelerations will be low-pass filtered using a bi-directional fourth order Butterworth filter with a cut-off frequency of $30 \mathrm{~Hz}{ }^{56}$ Measurements derived from the accelerometry data will include: (1) peak acceleration (root mean square (RMS)) and (2) HR, both of which will be calculated for the AP, ML and vertical (VT) axes of the head and trunk accelerometers separately. The HR has been used previously to evaluate dynamic postural instability in people with $\mathrm{PD}^{15} 37$ and will be used in this study to provide an indication of how 
Table 1 The primary, secondary and tertiary outcomes measures and the time points at which they will be assessed during the study

\begin{tabular}{|c|c|c|c|c|}
\hline & Outcome measures & $\begin{array}{l}\text { Baseline } \\
\text { (week 0) }\end{array}$ & $\begin{array}{l}\text { Postintervention } \\
\text { (week 12) }\end{array}$ & $\begin{array}{l}\text { Final } \\
\text { assessment } \\
\text { (week 24) }\end{array}$ \\
\hline \multicolumn{5}{|l|}{ Primary outcome measure } \\
\hline Dynamic postural stability & Harmonic ratio (AP, ML, VT) & $\mathrm{x}$ & $x$ & $x$ \\
\hline \multicolumn{5}{|l|}{ Secondary outcome measures } \\
\hline \multirow[t]{2}{*}{ Static postural stability } & Peak RMS displacement (AP, ML) & $\mathrm{x}$ & $\mathrm{x}$ & $x$ \\
\hline & Sway velocity (AP, ML) & $x$ & $x$ & $x$ \\
\hline $\begin{array}{l}\text { Bilateral trunk muscle } \\
\text { function }\end{array}$ & $\begin{array}{l}\text { Peak RMS activity (ES at T10 and L3 } \\
\text { levels) }\end{array}$ & $x$ & $x$ & $x$ \\
\hline \multicolumn{5}{|l|}{ Tertiary outcome measures } \\
\hline \multirow[t]{5}{*}{ Disease severity } & UPDRS III & $\mathrm{x}$ & $\mathrm{x}$ & $\mathrm{x}$ \\
\hline & FOGQ & $x$ & $x$ & $x$ \\
\hline & ABC scale & $\mathrm{x}$ & $\mathrm{x}$ & $\mathrm{x}$ \\
\hline & $\begin{array}{l}\text { Schwab and England Activities of daily } \\
\text { living }\end{array}$ & $x$ & $x$ & $x$ \\
\hline & PDQ-39 & $x$ & $x$ & $x$ \\
\hline \multirow[t]{4}{*}{ Other variables } & Intervention compliance & $\mathrm{x}$ & $\mathrm{x}$ & $\mathrm{x}$ \\
\hline & Adverse events & $x$ & $x$ & $x$ \\
\hline & Daily levodopa equivalents & $\mathrm{x}$ & $\mathrm{x}$ & $x$ \\
\hline & International physical activity questionnaire & & $x$ & $x$ \\
\hline \multicolumn{5}{|l|}{ Screening measures } \\
\hline Cognitive function & Addenbrooke's cognitive examination & $x$ & & \\
\hline Visual function & Bailey-Lovie high-contrast visual acuity & $\mathrm{x}$ & & \\
\hline
\end{tabular}

well the movement patterns of the head and trunk are controlled during normal gait.

Raw EMG data will be high-pass filtered at $100 \mathrm{~Hz}$ to remove heart rate artefact from the signal and then fullwave rectified and low-pass filtered (4th order Butterworth filter) at $20 \mathrm{~Hz}{ }^{57}$ Following filtering of the data, the RMS of the muscle activity throughout the walking trials will be calculated over a $50 \mathrm{~ms}^{57}$ moving average window, with a $25 \mathrm{~ms}$ overlap. ${ }^{55}$ To facilitate comparisons between participants and across testing days, the activation levels of the trunk muscles will be normalised to the peak RMS amplitude of the muscle activity recorded during the MVC trials. The peak normalised RMS muscle activities derived from three complete gait cycles for each leg from each of the four trials $(\mathrm{n}=12$ gait cycles per leg) will then be averaged and these data will be used for all subsequent analyses.

\section{Randomisation and blinding}

After completion of the baseline assessments, participants will be randomised using a computerised random number generator (block size $=3$ ) in a 1:1:1 ratio to one of the three intervention groups: (1) exercise 1 day/ week, (2) exercise 3 days/week or (3) education. To minimise the possibility of introducing issues related to interrater reliability and/or biasing the outcomes, the clinical assessments will be conducted by an individual who is trained to administer the tests, but who will not be involved with the recruitment and allocation of participants to intervention groups and will also be blinded to intervention status. Furthermore, another member of the research team responsible for processing and analysing the data related to the assessment of static and dynamic postural stability will recruit and assign participants to intervention groups, however will be blinded to the group allocation of the participants during data analysis.

\section{Intervention}

At baseline, all participants will receive a $10-15 \mathrm{~min}$ one-off presentation outlining the evidence that supports exercise as an effective means of improving movement and postural stability in people with PD. Participants in the education group will be encouraged to continue their day-to-day lives, as usual, but will receive a weekly multidisciplinary education package that will include a health tip that will explain how, for example, exercise, nutrition and/or sleep quality may influence their falls risk and quality of life. The education group represents what would normally be seen in everyday life, with the education brochures created using scientific evidence drawn from pre-existing research and freely-available information sheets produced by government and not-for-profit organisations.

Participants assigned to the exercise groups will complete a low-level supervision, 12-week exercise programme aimed at improving trunk mobility and 
Figure 1 Study outline. Flow chart depicting the order of recruitment and testing procedures for the outlined study.

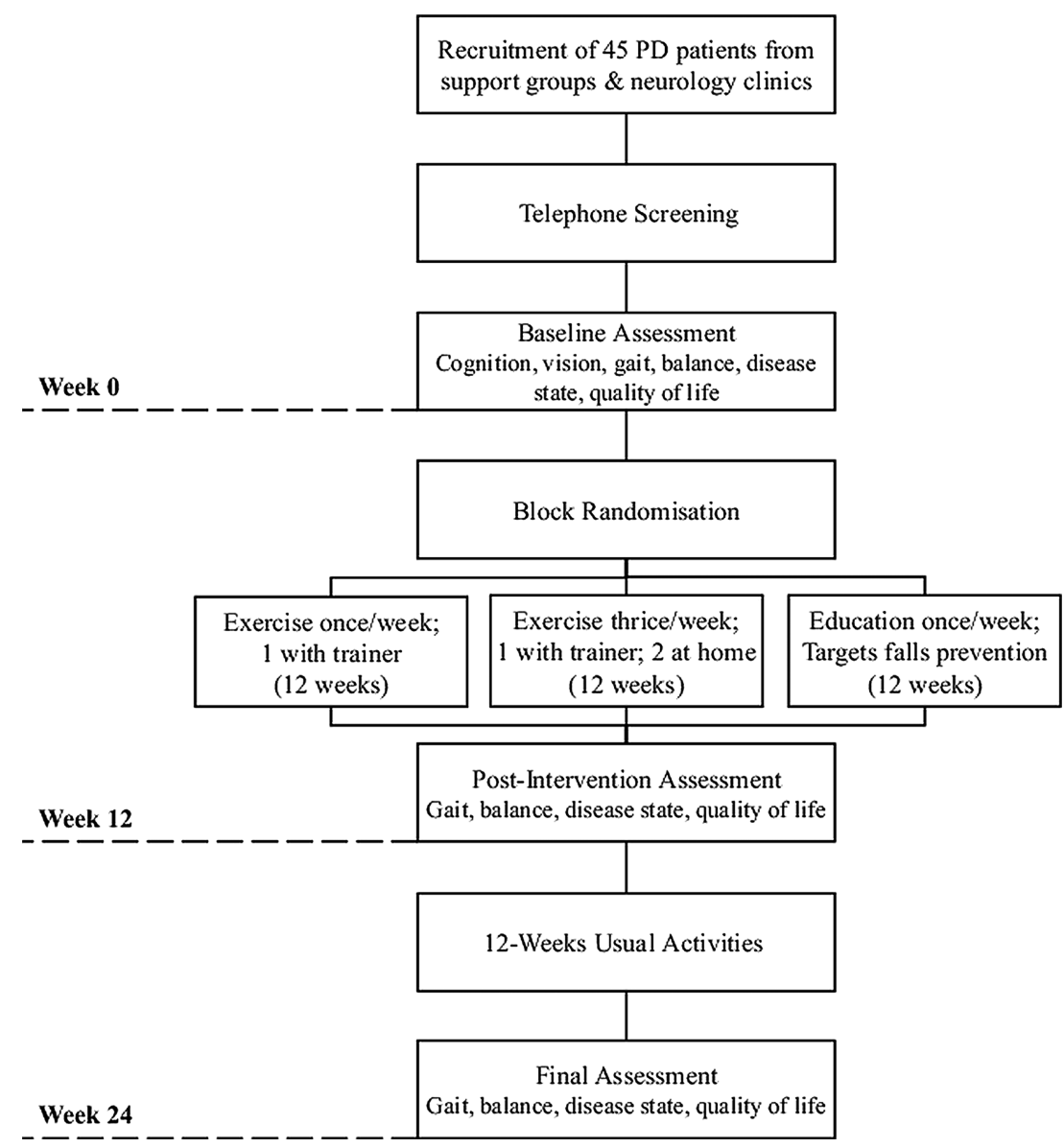

endurance, which will involve one supervised session each week with a trained Exercise Scientist at the University. The group exercising once per week will receive the intervention during the weekly supervised session, while the group exercising three times per week will be asked to complete the protocol at home on two other days of the week, for a total of three training days per week. The exercise programme consists primarily of exercises that have previously been used in two different exercise-based interventions involving older adults ${ }^{58}$ and people with $\mathrm{PD},{ }^{59}$ that focused on improving trunk muscle strength and endurance. Importantly, the programme was designed to conform to the current recommendations for best clinical practice with respect to the implementation of exercise-based interventions for improving postural stability. ${ }^{27} 3360$ Specifically, the programme includes movements focusing on improving trunk mobility, exercises that target muscular strength and endurance, tasks that aim to develop balance under challenging situations (ie, on an unstable surface) and ambulating over different terrains in a real-world environment. The programme will progress in complexity to accommodate individuals with different physical capabilities. The primary movements used for the programme are outlined in table 2. Hold times for the endurance exercises begin at $5 \mathrm{~s}$ and repetitions begin at 10 or as many as achievable by the participant. In addition, as the participant progresses in the programme, a round and flat air filled disc will be incorporated to create an unstable surface and create a balance challenging environment during the exercises. For the walking portion of the programme, this will be completed on an outdoor walking path that specifically incorporates varying degrees of incline and decline, stairs and multiple surface types to simulate walking during activities of daily living. The various challenges offered by this walking course will serve to improve the participants' capacity to safely and effectively ambulate in predictable and unstable real-world environments.

To facilitate monitoring of activity levels during the 12-week intervention and the 12-week sustainability periods, all participants will be asked to record their weekly activity levels using the International Physical Activity Questionnaire (IPAQ ${ }^{61}$ during these periods. The IPAQ is a questionnaire that has been shown to be both a valid and reliable tool for quantifying activity levels in different populations. ${ }^{62}{ }^{63}$ In addition, compliance to the intervention protocol and any adverse events will also be monitored and reported by the researchers.

\section{Statistical analysis}

Continuous data will first be checked for normal distribution and, where applicable, $\log$ transformation will be applied to the data. To assess for any significant differences between the groups with respect to the continuous demographic variables (eg, age, height, weight,) a one way 
Table 2 Summary of the specific tasks, repetitions and progressions for each of the exercises

\begin{tabular}{lll}
\hline Task & Movement & Repetitions/progression \\
\hline $\begin{array}{l}\text { Trunk mobility } \\
\text { Warm-up }\end{array}$ & Lateral bends & 10 to the left \\
& Torso totations & 10 to the right \\
& & 10 to the left \\
& Small arm circles & 10 to the right \\
& & 10 forward \\
& Large arm circles & 10 backward \\
& & 10 forward \\
& Torso rotations with high and low reaching & 10 backward \\
Trunk & Abdominal hollowing & 10 reaching up to left, down to right \\
endurance & Side bridging & 10 reaching up to right, down to left \\
& Front bridging & Increase difficulty of exercise by: \\
Mobility & Bird dog & Increased hold times \\
& Walking over surfaces of varying incline/decline, density and up & Movement complexity \\
Active cool & and down stairs & Introduce unstable support surface \\
down & Hamstring stretch & walking path \\
& Quadriceps stretch & 2 sets of $20 \mathrm{~s}$ holds \\
& Gastrocnemius/soleus stretch & 2 sets of $20 \mathrm{~s}$ holds \\
& Triceps stretch & 2 sets of $20 \mathrm{~s}$ holds \\
& Pectoral stretch & 2 sets of $20 \mathrm{~s}$ holds \\
& & 2 sets of $20 \mathrm{~s}$ holds
\end{tabular}

analysis of variance (ANOVA) will be used, while the $\chi^{2}$ test will be used to identify any significant differences in the frequency of categorical data (eg, gender, Hoehn \& Yahr scale). If a significant difference is found from the ANOVA, the Tukey's honestly significant difference test will be used to perform post hoc comparisons among the three groups. If the assumptions of normality (Shapiro-Wilks test) or homogeneity of variance (Levene's test) are still violated after log transformation, the nonparametric Kruskal-Wallis testing will replace the ANOVA. Analysis of the outcome measures for static and dynamic postural stability will be based on intention to treat principles. To assess the acute (12 weeks) and long-term (24 weeks) effects of the intervention on measures of postural stability, a repeated measures analysis of covariance (RM-ANCOVA) will be conducted, with the baseline value for each outcome measure and disease severity entered as covariates. To determine covariates, variables of age and disease severity will be graphed in relation to baseline measures of postural stability to identify any linear relationships. All statistical analyses will be completed in the Statistical Package for the Social Sciences (SPSS V.21.0) and the level of significance will be set at $\mathrm{p}<0.05$.

\section{DISCUSSION}

For people with PD, the increased risk of falls and fallrelated injuries has the potential to significantly influence an individual's psychological, physiological and socioeconomic state; ultimately impacting their quality of life. Although oral medications are known to improve many of the motor and non-motor symptoms associated with PD, late-stage symptoms such as gait difficulties and postural instability are not always responsive to this therapeutic intervention. ${ }^{64}$ As postural instability and gait difficulties contribute significantly to the high risk of falls in patients with PD, there is a strong need for further research examining additional non-invasive interventions that target the improvement of segmental control and postural alignment in this population.

To date, a number of studies have demonstrated that an exercise intervention can improve strength, ${ }^{65} 66$ measures of static postural stability ${ }^{67}$ and motor symptoms $^{17} 2868$ in people with PD. In contrast, a separate study reported no significant improvements in selfreported disability or clinical measures of balance, mobility or quality of life for people with PD following a 6-week home-based exercise intervention. ${ }^{18}$ Although these clinical tests have been widely used to assess falls risk in people with $\mathrm{PD}$, they may lack the sensitivity to provide real insight into the falls risk of this population. Specifically, it has been shown that the Tinetti Balance and Gait Assessment, Berg Balance Scale, Timed Up and Go, Functional Reach and Physiological Profile Assessment (PPA) of falls risk achieve only moderate sensitivities $(65-69 \%)$, specificities $(62-69 \%)$ and accuracies (53-68\%) when predicting prospective falls for people with PD. ${ }^{50}$ Continuous biomechanical measures, such as those provided by force platforms and accelerometers may help to resolve this problem by increasing the sensitivity of outcome measures to more accurately detect changes in motor performance.

From the perspective of maintaining balance, the trunk is believed to play an important role in maintaining head stability during dynamic tasks. During walking, forces are transmitted upwards from the feet following heel contact, which requires the legs, trunk and neck to act as shock absorbers to attenuate the load and maintain smooth 
movement patterns for the head. ${ }^{5}$ However, individuals with PD are known to have deficits in trunk control and trunk muscle function, ${ }^{53}$ which may impair their capacity to perform this role and increase their risk of falling. The findings of previous research tend to support this notion, indicating that people with PD who fall have greater ML head movement while walking on firm $^{1}$ and compliant ${ }^{16}$ surfaces and poorer pelvic control ${ }^{15}$ during unconstrained gait. As such, interventions aimed at improving trunk muscle functioning may help to improve postural stability and reduce falls for individuals with PD.

The intervention for this study was specifically developed to achieve this goal and will incorporate a series of safe and progressive exercises that were adapted from two previous studies examining the effects of exercise on balance and trunk muscle performance. The findings of these studies demonstrated that progressive exercises targeting improvements in the function of the deeper trunk muscles were effective in improving clinical measures of balance in older women who were at a high risk of falling. ${ }^{58}$ Similar exercises, when combined with aerobic exercises and stretching, were shown to significantly improve the strength and mobility of the trunk muscles in individuals with PD, but the authors did not report whether these improvements were associated with any changes in postural stability. ${ }^{59}$

As with any study of this nature, there are a number of limitations that have the potential to influence the outcomes of the proposed exercise-based intervention. First, to ensure the comfort and safety of the participants throughout the data collection and exercise (if applicable) sessions, participants will complete the baseline, follow-up and training sessions while on-medication. As such, it is possible that dopamine-induced side effects of the medication may influence their performances on some of the laboratory and/or clinical assessments. However, details regarding medications will be collected and participants will be asked to report any changes in medications during the study period. If differences are identified between the groups with respect to disease duration, disease severity or medications, these variables will be entered as covariates in the statistical model. Second, the sample size for this study may seem small compared with other studies that have used exercise-based interventions to reduce falls in older adults ${ }^{69}$ or people with PD. ${ }^{18}$ However, as supported by the presented power calculation, the target sample size of 15 participants per group is adequate to detect differences in our chosen primary outcome measure and will accommodate an attrition rate of $25 \%$.

In conclusion, there is a growing body of evidence to suggest that regular exercise has the potential to reduce the risk of falling in people with $\mathrm{PD}^{17}$ and may even help to reduce the number of falls experienced by some individuals. ${ }^{18}$ This study will be the first to examine whether a 12-week training programme aimed at improving trunk mobility and endurance has the potential to improve measures of postural stability in this population. If found to be effective, this training programme will provide a safe and inexpensive exercise-based therapy option that will help to maintain and/or improve postural stability and ultimately contribute to improving quality of life for people with Parkinson's disease.

Contributors RPH and MHC designed the study, obtained funding and completed extensive preparation to develop the study protocol. MHC will oversee the execution of the study and will be responsible for administering the clinical tests and assisting with recruitment of participants. RPH will be responsible for the day-to-day management of the study, data collection, data analysis and interpretation of the findings. GAN provided important assistance with the development of the study protocol and will be responsible for participant allocation. PAS will be involved in assisting with participant recruitment and with the interpretation of the clinical relevance of the study's outcomes. RPH and MHC developed the initial draft of this manuscript and all authors contributed to the refinement and finalisation of the submitted manuscript.

Funding This project is supported by research funding provided by the Australian Catholic University (grant number 2013000584). Furthermore, Dr Michael H. Cole was supported by an Australian National Health and Medical Research Council Early Career Research Fellowship (grant number 1006481).

Competing interests None.

Ethics approval Australian Catholic University Human Research Ethics Committee.

Provenance and peer review Not commissioned; externally peer reviewed.

Open Access This is an Open Access article distributed in accordance with the Creative Commons Attribution Non Commercial (CC BY-NC 4.0) license, which permits others to distribute, remix, adapt, build upon this work noncommercially, and license their derivative works on different terms, provided the original work is properly cited and the use is non-commercial. See: http:// creativecommons.org/licenses/by-nc/4.0/

\section{REFERENCES}

1. Cole MH, Silburn PA, Wood JM, et al. Falls in Parkinson's disease: kinematic evidence for impaired head and trunk control. Mov Disord 2010;25:2369-78

2. Wood B, Bilclough JA, Bowron A, et al. Incidence and prediction of falls in Parkinson's disease: a prospective multidisciplinary study. J Neurol Neurosurg Psychiatry 2002;72:721-5.

3. Grimbergen YA, Munneke M, Bloem BR. Falls in Parkinson's disease. Curr Opin Neurol 2004;17:405-15.

4. Bloem BR, Hausdorff JM, Visser JE, et al. Falls and freezing of gait in Parkinson's disease: a review of two interconnected, episodic phenomena. Mov Disord 2004:19:871-84.

5. Kavanagh J, Barrett R, Morrison S. The role of the neck and trunk in facilitating head stability during walking. Exp Brain Res 2006;172: 454-63.

6. Wright W, Gurfinkel VS, Nutt J, et al. Axial hypertonicity in Parkinson's disease: direct measurements of trunk and hip torque. Exp Neurol 2007;208:38-46.

7. Clark S, Rose DJ. Evaluation of dynamic balance among community-dwelling older adult fallers: a generalizability study of the limits of stability test. Arch Phys Med Rehabil 2001;82:468-74.

8. Goldberg A, Hernandez ME, Alexander NB. Trunk repositioning errors are increased in balance-impaired older adults. J Gerontol A Biol Sci Med Sci 2005;60:1310-14.

9. Grabiner MD, Donovan S, Bareither ML, et al. Trunk kinematics and fall risk of older adults: translating biomechanical results to the clinic. $J$ Electromyogr Kinesiol 2008;18:197-204.

10. Winter DA. Biomechanics and motor control of human movement. John Wiley \& Sons, 2009.

11. Cromwell R, Aadland-Monahan TK, Nelson AT, et al. Sagittal plane analysis of head, neck, and trunk kinematics and electromyographic activity during locomotion. J Orthop Sports Phys Ther 2001;31:255.

12. Smidt GL, Arora JS, Johnston RC. Accelerographic analysis of several types of walking. Am J Phys Med Rehabil 1971;50:285-300.

13. Menz HB, Lord SR, Fitzpatrick RC. Acceleration patterns of the head and pelvis when walking on level and irregular surfaces. Gait Posture 2003;18:35-46.

14. Van der Burg J, van Wegen EEH, Rietberg MB, et al. Postural control of the trunk during unstable sitting in Parkinson's disease. Parkinsonism Relat Disord 2006;12:492-8. 
15. Latt MD, Lord SR, Morris JGL, et al. Clinical and physiological assessments for elucidating falls risk in Parkinson's disease. Mov Disord 2009;24:1280-9.

16. Cole MH, Silburn PA, Wood JM, et al. Falls in Parkinson's disease: evidence for altered stepping strategies on compliant surfaces. Parkinsonism Relat Disord 2011;17:610-16.

17. Allen NE, Canning CG, Sherrington $\mathrm{C}$, et al. The effects of an exercise program on fall risk factors in people with Parkinson's disease: a randomized controlled trial. Mov Disord 2010;25:1217-25.

18. Ashburn A, Fazakarley L, Ballinger $\mathrm{C}$, et al. A randomised controlled trial of a home based exercise programme to reduce the risk of falling among people with Parkinson's disease. J Neurol Neurosurg Psychiatry 2007;78:678-84.

19. Ebersbach G, Edler D, Kaufhold O, et al. Whole body vibration versus conventional physiotherapy to improve balance and gait in Parkinson's disease. Arch Phys Med Rehabil 2008;89:399-403.

20. Morris M, Menz HB, McGinley JL, et al. Falls and mobility in Parkinson's disease: protocol for a randomised controlled clinical trial. BMC Neurol 2011;11:93.

21. Morris ME, Martin C, McGinley JL, et al. Protocol for a home-based integrated physical therapy program to reduce falls and improve mobility in people with Parkinson's disease. BMC Neurol 2012;12:54

22. Barnett A, Smith B, Lord SR, et al. Community-based group exercise improves balance and reduces falls in at-risk older people: a randomised controlled trial. Age Ageing 2003;32:407-14.

23. Rochester L, Baker K, Hetherington V, et al. Evidence for motor learning in Parkinson's disease: acquisition, automaticity and retention of cued gait performance after training with external rhythmical cues. Brain Res 2010;1319:103-11.

24. Fox KR. The influence of physical activity on mental well-being Public Health Nutr 1999:2:411-18.

25. Cooney GM, Dwan K, Greig CA, et al. Exercise for depression. Cochrane Database Syst Rev 2013;9:CD004366.

26. Gillespie LD, Robertson MC, Gillespie WJ, et al. Interventions for preventing falls in older people living in the community. Cochrane Database Syst Rev 2012;9:CD007146.

27. Sherrington C, Whitney JC, Lord SR, et al. Effective exercise for the prevention of falls: a systematic review and meta-analysis. $J \mathrm{Am}$ Geriatr Soc 2008:56:2234-43.

28. Lun V, Pullan N, Labelle N, et al. Comparison of the effects of a self-supervised home exercise program with a physiotherapistsupervised exercise program on the motor symptoms of Parkinson's disease. Mov Disord 2005;20:971-5.

29. Tomlinson CL, Patel S, Meek C, et al. Physiotherapy intervention in Parkinson's disease: systematic review and meta-analysis. $B M J$ 2012;345:e5004.

30. Tomlinson CL, Patel S, Meek C, et al. Physiotherapy versus placebo or no intervention in Parkinson's disease. Cochrane Database Syst Rev 2012;8:CD002817.

31. Oguh O, Eisenstein A, Kwasny M, et al. Back to the basics: regular exercise matters in Parkinson's disease: results from the National Parkinson Foundation registry study. Parkinsonism Relat Disord 2014:20:1221-5.

32. Murray DK, Sacheli MA, Eng JJ, et al. The effects of exercise on cognition in Parkinson's disease: a systematic review. Trans/ neurodegen 2014;3:5.

33. Li F, Harmer P, Fitzgerald K, et al. Tai chi and postural stability in patients with Parkinson's disease. N Engl J Med 2012;366:511-19.

34. Tomlinson CL, Herd CP, Clarke CE, et al. Physiotherapy for Parkinson's disease: a comparison of techniques. Cochrane Database Syst Rev 2014;6:CD002815.

35. Schulz KF, Altman DG, Moher D. CONSORT 2010 statement: updated guidelines for reporting parallel group randomised trials. BMC Med 2010;8:18.

36. Hughes AJ, Daniel SE, Kilford L, et al. Accuracy of clinical diagnosis of idiopathic Parkinson's disease: a clinico-pathological study of 100 cases. J Neurol Neurosurg Psychiatry 1992;55:181-4.

37. Lowry KA, Smiley-Oyen AL, Carrel AJ, et al. Walking stability using harmonic ratios in Parkinson's disease. Mov Disord 2009;24:261-7.

38. Mathuranath PS, Nestor PJ, Berrios GE, et al. A brief cognitive test battery to differentiate Alzheimer's disease and frontotemporal dementia. Neurology 2000;55:1613-20.

39. Bailey IL, Lovie J. New design principles for visual acuity letter charts. Am J Optom Physiol Opt 1976:53:740.

40. Hoehn MM, Yahr MD. Parkinsonism: onset, progression, and mortality. Neurology 1998;50:318.

41. Schwab RS, England AC. Projection technique for evaluating surgery in Parkinson's disease. Third symposium on Parkinson's disease. Edinburgh: Livingstone, 1969.
42. Giladi N, Shabtai H, Simon ES, et al. Construction of freezing of gait questionnaire for patients with Parkinsonism. Parkinsonism Relat Disord 2000;6:165-70.

43. Powell LE, Myers AM. The activities-specific balance confidence (ABC) scale. J Gerontol A Biol Sci Med Sci 1995;50:M28-34.

44. Podsiadlo D, Richardson S. The timed "Up \& Go": a test of basic functional mobility for frail elderly persons. J Am Geriatr Soc 1991;39:142.

45. Peto V, Jenkinson C, Fitzpatrick R, et al. The development and validation of a short measure of functioning and well being for individuals with Parkinson's disease. Qual Life Res 1995;4:241-8.

46. Goetz CG, Poewe W, Rascol O, et al. Movement Disorder Society Task Force report on the Hoehn and Yahr staging scale: status and recommendations The Movement Disorder Society Task Force on rating scales for Parkinson's disease. Mov Disord 2004;19:1020-8.

47. Lovie-Kitchin JE. Validity and reliability of viscual acuity measurements. Ophthal Physiol Opt 1988;8:363-70.

48. Martinez-Martin P, Gil-Nagel A, Morlán Gracia L, et al. Intermediate scale for assessment of Parkinson's disease. Characteristics and structure. Parkinsonism Relat Disord 1995;1:97-102.

49. Ramaker C, Marinus J, Stiggelbout AM, et al. Systematic evaluation of rating scales for impairment and disability in Parkinson's disease. Mov Disord 2002;17:867-76.

50. Kerr G, Worringham CJ, Cole $\mathrm{MH}$, et al. Predictors of future falls in Parkinson disease. Neurology 2010;75:116-24.

51. Moe-Nilssen R. A new method for evaluating motor control in gait under real-life environmental conditions. Part 2: gait analysis. Clin Biomech 1998;13:328-35.

52. Winter D, Yack H. EMG profiles during normal human walking: stride-to-stride and inter-subject variability. Electroencephalogr Clin Neurophysiol 1987;67:402-11.

53. Bridgewater KJ, Sharpe $\mathrm{MH}$. Trunk muscle performance in early Parkinson's disease. Phys Ther 1998;78:566-76.

54. Hewson D, Hogrel J-Y, Langeron Y, et al. Evolution in impedance at the electrode-skin interface of two types of surface EMG electrodes during long-term recordings. J Electromyogr Kinesiol 2003;13:273-9.

55. Vera-Garcia FJ, Moreside JM, McGill SM. MVC techniques to normalize trunk muscle EMG in healthy women. J Electromyogr Kinesiol 2010;20:10-16.

56. Kavanagh J, Barrett R, Morrison S. Upper body accelerations during walking in healthy young and elderly men. Gait Posture 2004;20:291-8.

57. Robichaud JA, Pfann KD, Comella CL, et al. Effect of medication on EMG patterns in individuals with Parkinson's disease. Mov Disord 2002:17:950-60.

58. Hesari AF, Mahdavi S, Abadi MR, et al. Comparisons of berg balance scale following core stabilization training in women elderly. Ann Biol Res 2012;3:1499-504.

59. Bridgewater KJ, Sharpe MH. Trunk muscle training and early Parkinson's disease. Physiother Theory Pract 1997;13:139-53.

60. Smania N, Corato $\mathrm{E}$, Tinazzi M, et al. Effect of balance training on postural instability in patients with idiopathic Parkinson's disease. Neurorehabil Neural Repair 2010;24:826-34.

61. Craig CL, Marshall Al, Söjöstrom M, et al. International physical activity questionnaire: 12 -country reliability and validity. $\mathrm{Med} \mathrm{SCl}$ Sports Exerc 2003;35:1381-95.

62. Hagstromer M, Oja P, Sjostrom M. The International Physical Activity Questionnaire (IPAQ): a study of concurrent and construct validity. Public Health Nutr 2006;9:755-62.

63. Hallal PC, Victora CG. Reliability and validity of the International Physical Activity Questionnaire (IPAQ). Med Sci Sports Exerc 2004;36:556.

64. Ferraye MU, Debu B, Fraix V, et al. Effects of pedunculopontine nucleus area stimulation on gait disorders in Parkinson's disease. Brain 2010;133:205-14

65. Crizzle AM, Newhouse IJ. Is physical exercise beneficial for persons with Parkinson's disease? Clin J Sport Med 2006;16:422-5.

66. Goodwin VA, Richards SH, Taylor RS, et al. The effectiveness of exercise interventions for people with Parkinson's disease: a systematic review and meta-analysis. Mov Disord 2008;23:631-40.

67. Hirsch MA, Toole T, Maitland CG, et al. The effects of balance training and high-intensity resistance training on persons with idiopathic Parkinson's disease. Arch Phys Med Rehabil 2003;84:1109-17.

68. Jöbges EM, Spittler-Schneiders H, Renner CIE, et al. Clinical relevance of rehabilitation programs for patients with idiopathic Parkinson syndrome. II: symptom-specific therapeutic approaches. Parkinsonism Relat Disord 2007:13:203-13.

69. Kyrdalen IL, Moen K, Røysland AS, et al. The Otago Exercise Program performed as group training versus home training in 